\title{
ELASTICITY ANISOTROPY OF COMPOSITES
}

\author{
Vladimir Glukhikh
}

Saint Petersburg State University of Architecture and Civil Engineering

Vtoraja Krasnoarmejskaja ul., 4, St. Petersburg, 190005, Russia

VNGlukhikh@mail.ru

\begin{abstract}
The article presents a solution to a problem on the establishment of mathematical relations between elastic constants of anisotropic fiber composites in principal anisotropy directions. A stress function in the form of a polynomial sum was used to solve the fourth-order differential equation in partial derivatives for an orthotropic body, thus allowing determining elastic constants of fiber composites with different directions of reinforcing fibers by means of mathematical calculations. It was established that all anisotropic materials could be conditionally divided into two groups.

One group includes composites in which elastic constants take an intermediate extreme value at $60^{\circ}$ upon change of the reinforcing fibers position from 0 to $90^{\circ}$. The other group includes composites with two extreme values - at 0 and $90^{\circ}$. This new knowledge obtained for the first time shall be used to provide rationale for the method of composite strength assessment by means of calculations depending on the position of reinforcing fibers, which was previously possible only upon execution of experimental studies.
\end{abstract}

\section{Keywords}

Composite materials, elastic constants, anisotropy, fiber materials, filament-wound materials, principal directions, orthotropic body.

\section{Introduction}

Evolution of living systems and vegetable origin systems is accompanied by optimization of their "engineering" components with the following improvement of resistance to external impacts. An ordered structure (17) forms under the influence of the environment.

The strongest reinforcing fibers formed in mechanical tissues during evolutionary optimization of bearing structures. The direction of such fibers coincides with maximum tensile and compressive stresses.

Micro- and macro-structure orientation providing high resistance of mechanical tissues to external impacts represents a result of evolutionary optimization of support systems in nature, including bone tissues of animals and humans, plant and tree stems. In recent decades, such ordered structure of mechanical tissues in the plant, animal and human world has become an object of increasing attention with the purpose of borrowing when developing new high-strength composites for engineering and construction - materials with pre-defined properties.

In a relatively short period of time, composites have evolved from materials designed exclusively for strategic and military use to wide application materials used in various industries and national economy sectors.

Due to the increasing demand of population in many countries for the wide range of residential buildings, special attention is given to application of new advanced high-performance composites with high aesthetic, as well as special characteristics, intended to be used in construction. 
For more than one decade, composites with a structure reminding the tree stem have been made by winding (with the use of reinforcement made of fiber metal and nonmetal materials in the form of fibers or strips (16)).

In many cases, filament-wound composites can be referred to cylindrically anisotropic materials. The full set of characteristics of their elastic stress-strain behavior includes nine independent elastic constants (Ashkenazi, 1978; Lekhnitsky, 1977).

Knowing values of elastic constants in principal anisotropy directions, we can determine the value of any elastic constant in the required direction, which is often necessary when determining deformations in directions not coinciding with principal ones (Ashkenazi, 1978; Ashkenazi et al., 1981; Lekhnitsky, 1957).

To determine values of elastic constants in any directions, we can use equations suggested by S.G. Lekhnitsky (1957), Ye.K. Ashkenazi (1978, 1981), A.N. Mitinsky $(1948,1949)$ and other scientists. Those equations include experimentally found elastic constants in principal anisotropy directions. The authors also paid special attention to the inclusion of the shear and elastic moduli, as well as the Poisson's ratio in directions at the angle of $45^{\circ}$ in relation to reinforcing fibers, the method of determination of which is imperfect, according to the authors. This is due to the lack of mathematical relations between elastic constants in principal anisotropy directions like in isotropic materials. This paper suggests a way to improve the method for determination of elastic constants in filament-wound composites by establishment of mathematical relations between their elastic constants in principal anisotropy directions.

\section{Research method}

As a basis of the theoretical research, a fourth-order differential equation in partial derivatives (known in the theory of elasticity of an anisotropic body) for a cylindrically anisotropic orthotropic body, in polar coordinates, which the stress function satisfies, was taken. Without consideration of body forces, this equation in polar coordinates is written as follows (Ashkenazi, 1978; Lekhnitsky, 1957, 1977):

$$
\begin{aligned}
& \frac{1}{E_{t}} \cdot \frac{\partial^{4} F}{\partial r^{4}}+\left(\frac{1}{G_{r t}}-\frac{2 \mu_{r t}}{E_{r}}\right) \cdot \frac{1}{r^{2}} \cdot \frac{\partial^{4} F}{\partial r^{2} \partial \theta^{2}}+\frac{1}{E_{r}} \cdot \frac{1}{r^{4}} \\
& \frac{\partial^{4} F}{\partial \theta^{4}}+\frac{2}{E_{t}} \cdot \frac{1}{r} \cdot \frac{\partial^{3} F}{\partial r^{3}}-\left(\frac{1}{G_{r t}}-\frac{2 \mu_{r t}}{E_{r}}\right) \cdot \frac{1}{r^{3}} \cdot \frac{\partial^{3} F}{\partial r \partial \theta^{2}}- \\
& -\frac{1}{E_{r}} \cdot \frac{1}{r^{2}} \cdot \frac{\partial^{2} F}{\partial r^{2}}+\left(2 \frac{1-\mu_{r t}}{E_{r}}+\frac{1}{G_{r t}}\right) \cdot \frac{1}{r^{4}} \cdot \frac{\partial^{2} F}{\partial \theta^{2}}+ \\
& +\frac{1}{E_{r}} \cdot \frac{1}{r^{3}} \cdot \frac{\partial F}{\partial r}=0
\end{aligned}
$$

It is convenient to use the obtained equation for circular plates. To solve tasks related to design of rectangular elements, it is more convenient to use the last equation in Cartesian coordinates.

After differentiation and substitution of the corresponding derivatives in equation (1), the following fourth-order differential equation in partial derivatives in Cartesian coordinates is obtained for a cylindrically anisotropic orthotropic body:

$$
\begin{aligned}
& \frac{\partial^{4} F}{\partial x^{4}}\left(x^{4}+B x^{2} y^{2}+\alpha^{2} y^{4}\right)+\frac{\partial^{4} F}{\partial y^{4}}\left(y^{4}+B x^{2} y^{2}+\alpha^{2} x^{4}\right)+ \\
& +\frac{\partial^{4} F}{\partial y \partial x^{3}}\left[2 x^{2}+B\left(y^{2}-x^{2}\right)-2 \alpha^{2} y^{2}\right] \cdot 2 x y+\frac{\partial^{4} F}{\partial x \partial y^{3}} . \\
& {\left[2 y^{2}+B\left(x^{2}-y^{2}\right)-2 \alpha^{2} x^{2}\right] \cdot 2 x y+\frac{\partial^{4} F}{\partial x^{2} \partial y^{2}} \text {. }} \\
& \cdot\left[6 x^{2} y^{2}+B\left(x^{4}-4 x^{2} y^{2}+y^{4}\right)+6 \alpha^{2} x^{2} y^{2}\right]+\frac{\partial^{3} F}{\partial x^{3}} \\
& \cdot\left[2 x^{2}+B\left(3 y^{2}-x^{2}\right)-6 \alpha^{2} y^{2}\right] \cdot x+\frac{\partial^{3} F}{\partial y^{3}} . \\
& \cdot\left[2 y^{2}+B\left(3 x^{2}-y^{2}\right)-6 \alpha^{2} x^{2}\right] \cdot y+\frac{\partial^{3} F}{\partial y \partial x^{2}} . \\
& \cdot\left[2 x^{2}\left(1+2 \alpha^{2}\right)+B\left(y^{2}-3 x^{2}\right)-2 \alpha^{2} y^{2}\right] \cdot 3 y+\frac{\partial^{3} F}{\partial x \partial y^{2}} \\
& \cdot\left[2 y^{2}\left(1+2 \alpha^{2}\right)+B\left(x^{2}-3 y^{2}\right)-2 \alpha^{2} x^{2}\right] \cdot 3 x+ \\
& +\frac{\partial^{2} F}{\partial x^{2}}\left(2 \alpha^{2}-B\right)\left(x^{2}-y^{2}\right)+\frac{\partial^{2} F}{\partial y^{2}}\left(B-2 \alpha^{2}\right) \text {. } \\
& \cdot\left(x^{2}-y^{2}\right)+\frac{\partial^{2} F}{\partial x \partial y}\left(2 \alpha^{2}-B\right) \cdot 4 x y=0 \\
& \text { where } \alpha^{2}=E_{t} / E_{r} ; B=E_{t}\left(\frac{1}{G_{r t}}-\frac{2 \mu_{r t}}{E_{r}}\right)
\end{aligned}
$$

If the load on edges and ends of a rectangular anisotropic plate is set in the form of an entire algebraic function, then the corresponding stress function can be taken in the form of an entire polynomial. The task is solved using the stress function in the form of a sum of polynomials (Kurdyumov, 1946; Glukhikh, 2001, 1997, 2007, 2008, 2009):

$$
F_{(x, y)}=\sum_{k=1}^{N} f_{k}(y) x^{k}
$$

where $f_{k}(y)-a$ function unknown at the initial solution stage, that shall satisfy differential equation (Ashkenazi, Ganov, 1981) and conditions on the plate contour.

The stress function is taken in the form of the following sum of polynomials (Kurdyumov, 1946; Glukhikh, 2001):

$$
F(x, y)=f_{0}(y)+x f_{1}(y)+x^{2} f_{2}(y)+x^{3} f_{3}(y)+x^{4} f_{4}(y)+
$$




$$
+x^{5} f_{5}(y)+x^{6} f_{6}(y)+x^{7} f_{7}(y)+x^{8} f_{8}(y)+x^{9} f_{9}(y)
$$

The corresponding derivatives of the stress function $F(x, y)$ are substituted in differential equation (2).

If we equate factors multiplying the corresponding $x$ degrees to zero, we will obtain differential equations that the selected stress function shall satisfy. The first group of equations will include functions $f_{k}(y)$ with even numbers only, including $f_{0}(y)$. The second group of equations will include functions $f_{k}(y)$ with odd numbers.

The first group of equations is as follows:

$$
\begin{aligned}
& 24 \alpha^{2} y^{4} f_{4}(y)+2 B y^{4} f_{2}^{\prime \prime}(y)+6\left(B-2 \alpha^{2}\right) y^{3} f_{2}^{\prime}(y)+ \\
& +2\left(B-2 \alpha^{2}\right) y^{2} f_{2}(y)+y^{4} f_{0}^{\mathrm{IV}}(y)+(2-B) y^{3} f_{0}^{\prime \prime \prime}(y)- \\
& -\left(B-2 \alpha^{2}\right) y^{2} f_{2}^{\prime \prime}(y)=0
\end{aligned}
$$$$
360 \alpha^{2} y^{4} f_{6}(y)+12 B y^{4} f_{4}^{\prime \prime}(y)+84\left(B-2 \varepsilon^{2}\right) y^{3} f_{4}^{\prime}(y)+
$$$$
+\left(108 B-168 \alpha^{2}\right) y^{2} f_{4}(y)+5(2-B) y^{3} f_{2}^{\prime \prime \prime}(y)+(24-
$$$$
\left.-27 B+38 \alpha^{2}\right) y^{2} f_{2}^{\prime \prime}(y)+\left(12+40 \alpha^{2}-26 B\right) y f_{2}^{\prime}(y)+
$$$$
-2\left(B-2 \alpha^{2}\right) f_{2}(y)+B y^{2} f_{2}^{\mathrm{IV}}(y)+3\left(B-2 \alpha^{2}\right) y f_{0}^{\prime \prime \prime}(y)+
$$$$
+\left(B-2 \alpha^{2}\right) f_{0}^{\prime \prime}(y)=0
$$$$
1680 \alpha^{2} y^{4} f_{8}(y)+30 B y^{4} f_{6}^{\prime \prime}(y)+330\left(B-2 \alpha^{2}\right) y^{3} f_{6}^{\prime}(y)+
$$$$
+\left(750 B-780 \alpha^{2}\right) y^{2} f_{6}(y)+y^{4} f_{4}^{\mathrm{IV}}(y)+9(2-B) y^{3} f_{4}^{\prime \prime \prime}(y)+
$$$$
+\left(96+122 \alpha^{2}-85 B\right) y^{2} f_{4}^{\prime \prime}(y)+\left(168-172 B+176 \alpha^{2}\right) y f_{4}^{\prime}(y)+
$$$$
+12\left(6-3 B-2 \alpha^{2}\right) f_{4}(y)+B y^{2} f_{2}^{I V}(y)+7\left(B-2 \alpha^{2}\right) y f_{2}{ }^{\prime \prime}(y)+
$$$$
+\left(9 B-14 \alpha^{2}\right) f_{2}^{\prime \prime \prime}(y)+\alpha^{2} f_{0}^{\mathrm{IV}}(y)=0
$$

When using stress function components $\left(f_{10}(y), f_{12}(y)\right.$, ...), equations following equation (8) form in the group, including $f_{10}(y), f_{12}(y)$, etc., respectively.

It should be noted that all functions newly appearing in equations (7), (8), etc. will be related to $f_{0}(y), f_{2}(y), f_{4}(y)$ as per equation (6).

The second group of equations is as follows: $120 \alpha^{2} y^{4} f_{5}(y)+6 B y^{4} f_{3}^{\prime \prime}(y)+30\left(B-2 \alpha^{2}\right) y^{3} f_{3}^{\prime}(y)+$ $+24\left(B-2 \alpha^{2}\right) y^{2} f_{3}(y)+y^{4} f_{1}^{\mathrm{IV}}(y)+3(2-B) y^{3} f_{1}^{\prime \prime \prime}(y)+$ $+2\left(3+7 \alpha^{2}-5 B\right) y^{2} f_{1}^{\prime \prime}(y)-4\left(B-2 \alpha^{2}\right) y f_{1}^{\prime}(y)=0$;

$840 \alpha^{2} y^{4} f_{7}(y)+20 B y^{4} f_{5}^{\prime \prime}(y)+180\left(B-2 \alpha^{2}\right) y^{3} f_{5}^{\prime}(y)+$ $\left(320 B-400 \alpha^{2}\right) y^{2} f_{5}(y)+y^{4} f_{3}^{I V}(y)+7(2-B) y^{3} f_{3}^{\prime \prime}(y)$ $+\left(54-52 B+74 \alpha^{2}\right) y^{2} f_{3}^{\prime \prime}(y)+\left(60+96 \alpha^{2}-78 B\right) y f_{3}^{\prime}(y)+$ $+121+\alpha^{2}-B() f_{3}(y)+B y^{2} f_{1}^{\mathrm{IV}}(y)+5\left(B-2 \alpha^{2}\right) y f_{1}^{\prime \prime \prime}(y)+$ $+4\left(B-2 \alpha^{2}\right) f_{1}^{\prime \prime}(y)=0$; $3024 \alpha^{2} y^{4} f_{9}(y)+42 B y^{4} f_{7}^{\prime \prime}(y)+\left(546 B-1092 \alpha^{2}\right) y^{3} f_{7}^{\prime}(y)+$ $+\left(1512 B-1344 \alpha^{2}\right) y^{2} f_{7}(y)+y^{4} f_{5}^{\mathrm{IV}}(y)+11(2-B) y^{3} f_{5}^{\prime \prime \prime}(y)+$ $+\left(150+182 \alpha^{2}-126 B\right) y^{2} f_{5}^{\prime \prime}(y)+\left(360+280 \alpha^{2}-320 B\right) y f_{5}^{\prime}(y)+$ $+\left(240+40 \alpha^{2}-80 B\right) f_{5}(y)+B y^{2} f_{3}^{\mathrm{IV}}(y)+9\left(B-2 \alpha^{2}\right) y f_{3}^{\prime \prime \prime}(y)+$ $+\left(16 B-20 \alpha^{2}\right) f_{3}^{\prime \prime}(y)+\alpha^{2} f_{1}^{\mathrm{IV}}(y)=0$;
Here the same pattern as in the previous case manifests itself. When using additional functions $f_{11}(y)$, $f_{13}(y)$, etc., differential equations following equation (11) form, including $f_{11}(y), f_{13}(y)$, etc., respectively.

All functions $f_{7}(y), f_{9}(y)$, etc. are dependent on functions $f_{1}(y), f_{3}(y)$ and $f_{5}(y)$ as per (9).

Thus, a task with any number of functions $f_{k}(y)$ can be solved in the following way: at the first stage, functions $f_{0}(y), f_{1}(y), \ldots, f_{5}(y)$ are determined based on equations (6) and (9), and then the remaining functions are determined.

Considering the order of derivatives $f_{k}^{(n)}(y)$ and degree of variable $y$, the solution of homogeneous differential equations (6)-(11) with variable coefficients can be represented in the form of algebraic functions:

$f_{k}(y)=C_{0}+C_{1} y+C_{2} y^{2}+C_{3} y^{3}+C_{4} y^{4}+\ldots$

where $C$ - integration constants.

Using the stress function $F(x, y)$ in the form of a (7) sum of polynomials for solution of differential equations (6)-(11), we can assume a relation between integration constants. This inevitably derives from the requirement for satisfaction of those differential equations with the selected function $F(\mathrm{x}, \mathrm{y})$.

Let us use, for example, the following stress function at the first stage:

$$
F(x, y)=f_{0}(y)+x^{2} f_{2}(y)+x^{4} f_{4}(y)+x^{6} f_{6}(y)+x^{8} f_{8}(y)
$$

and differential equation (8); in order for $F(x, y)$ to satisfy differential equation (8), it is required to accept the following:

$$
\begin{aligned}
& f_{8}(y)=C_{81}+C_{82} y+C_{83} y^{2}+C_{84} y^{3} \\
& f_{6}(y)=C_{61}+C_{62} y+C_{63} y^{2}+C_{64} y^{3}+C_{65} y^{4}+C_{66} y^{5} \\
& f_{4}(y)=C_{41}+C_{42} y+C_{43} y^{2}+C_{44} y^{3}+C_{45} y^{4}+C_{46} y^{5}+C_{47} y^{6}+C_{48} y^{7} \\
& f_{2}(y)=C_{21}+C_{22} y+C_{23} y^{2}+C_{24} y^{3}+C_{25} y^{4}+C_{26} y^{5}+ \\
& +C_{27} y^{6}+C_{28} y^{7}+C_{29} y^{8}+C_{210} y^{9} \\
& f_{0}(y)=C_{01}+C_{02} y+C_{03} y^{2}+C_{04} y^{3}+C_{05} y^{4}+C_{06} y^{5}+ \\
& +C_{07} y^{6}+C_{08} y^{7}+C_{09} y^{8}+C_{010} y^{9}+C_{011} y^{10}+C_{012} y^{11}
\end{aligned}
$$

Substituting these functions and their corresponding derivatives in differential equation (6), and equating factors multiplying the corresponding y degrees to zero,

(10) the following correlations between integration constants can be obtained:

$C_{21}=C_{03}$

$C_{22}=C_{04} \frac{3\left(B-1-\alpha^{2}\right)}{2\left(B-2 \alpha^{2}\right)} ;$ etc.

Performing similar rearrangements with the use of functions (14)-(18) and differential equation (7), new correlations between arbitrary constants can be obtained:

$C_{03}=C_{21}$ 


$$
C_{22}=-C_{04} \frac{6\left(B-2 \alpha^{2}\right)}{3+11 \alpha^{2}-7 B}
$$

Let us perform similar rearrangements with the use of equations (14)-(18) and (8).

A similar solution can be achieved for three differential equations (9), (10) and (11) involving functions with odd numbers $f_{1}(y), f_{3}(y), f_{5}(y), f_{7}(y)$, represented in the following form:

$$
\begin{aligned}
& f_{9}(y)=C_{91}+C_{92} y+C_{93} y^{2}+C_{94} y^{3} \\
& f_{7}(y)=C_{71}+C_{72} y+C_{73} y^{2}+C_{74} y^{3}+C_{75} y^{4}+C_{76} y^{5} \\
& f_{5}(y)=C_{51}+C_{52} y+C_{53} y^{2}+C_{54} y^{3}+ \\
& +C_{55} y^{4}+C_{56} y^{5}+C_{57} y^{6}+C_{58} y^{7} ; \\
& f_{3}(y)=C_{31}+C_{32} y+C_{33} y^{2}+C_{34} y^{3}+C_{35} y^{4}+ \\
& +C_{36} y^{5}+C_{37} y^{6}+C_{38} y^{7}+C_{39} y^{8}+C_{310} y^{9} \\
& f_{1}(y)=C_{11}+C_{12} y+C_{13} y^{2}+C_{14} y^{3}+C_{15} y^{4}+C_{16} y^{5}+ \\
& +C_{17} y^{6}+C_{18} y^{7}+C_{19} y^{8}+C_{110} y^{9}+C_{111} y^{10}+C_{112} y^{11}
\end{aligned}
$$

Substituting (23)-(27) to (9) and performing rearrangements, we will obtain the following:

$$
C_{12}=0
$$

according to the task condition for stresses, $C_{11}=0$ shall be taken:

$$
C_{31}=-C_{13} \frac{3+11 \alpha^{2}-7 B}{6\left(B-2 \alpha^{2}\right)}
$$

Similar correlations between integration constants can be obtained after rearrangement of equation (8) considering (23) $-(27)$ :

$$
C_{31}=-C_{13} \frac{2\left(B-2 \alpha^{2}\right)}{3\left(1+\alpha^{2}-B\right)}
$$

Analyzing equation (22) at this stage when the integration constants are unknown, we shall note that the constant $C_{04}$ does not become zero upon solution of bending problems. It is known that in case of an isotropic body, the law of normal stress distribution by crosssectional height upon bending is linear.

Elastic properties of isotropic bodies are defined by three characteristics, two of which are independent:

$$
G=\frac{E}{2(1+\mu)}
$$

As noted above, a similar correlation should exist for a cylindrically anisotropic body.

Upon solution of differential equation (2) in Cartesian coordinates with application of the stress function in the form of a sum of polynomials (4), it was established that correlations between integration constants depended on elastic constants. This holds in equation (3).

Correlations (20) and (22) between the constants $C_{22}$ and $\mathrm{C}_{04}$ come under notice.
It was noted above that the constant $C_{04}$ does not become zero, therefore, the constant $C_{22}$ is not equal to zero as well. Let us equate factors multiplying $C_{04}$ in the right parts of equations (20) and (22) to each other:

$$
\frac{3\left(B-1-\alpha^{2}\right)}{2\left(B-2 \alpha^{2}\right)}=-\frac{6\left(B-2 \alpha^{2}\right)}{3+11 \alpha^{2}-7 B}
$$

The same can be performed for factors multiplying the constants $C_{31}$ and $C_{13}$ in correlations (29) and (30).

As a result of the rearrangement, equation (32) takes the following form:

$$
B^{2}-\frac{2}{3}\left(5+\alpha^{2}\right) B-\frac{5}{3} \alpha^{4}+\frac{14}{3} \alpha^{2}+1=0
$$

where the roots of the equation are:

$$
\text { a) } B_{(1)}=\frac{1+5 \alpha^{2}}{3} \text {; b) } B_{(2)}=3-\alpha^{2}
$$

\section{Results}

Based on (3), the shear modulus can be calculated:

$G_{r t}=\frac{E_{t}}{B+2 \mu_{t r}}$

which, considering (35), will be represented by the following:

$$
\begin{aligned}
& B<2 ; G_{r t}=\frac{3 E_{t}}{1+5 \alpha^{2}+6 \mu_{t r}} \\
& B>2 ; G_{r t}=\frac{E_{t}}{3-\alpha^{2}+2 \mu_{t r}}
\end{aligned}
$$

In case of an isotropic body, the last two equations will take the form of equation (31).

Results of equation (33) roots determination under different values of $\alpha^{2}$ are presented in Table 1. This correlation is graphically represented in Figure 1.

Table 1. $B$ values at different $\alpha^{2}$ values

\begin{tabular}{|c|l|l|l|l|l|l|}
\hline$\alpha^{2}$ & 0.3 & 0.4 & 0.5 & 0.6 & 0.7 & 0.8 \\
\hline$B_{(1)}=\frac{1+5 \alpha^{2}}{3}$ & 0.8333 & 1 & 1.1667 & 1.3333 & 1.5 & 1.6667 \\
\hline$B_{(2)}=3-\alpha^{2}$ & 2.7 & 2.6 & 2.5 & 2.4 & 2.3 & 2.2 \\
\hline
\end{tabular}

Several differences between calculation results and some data (Ashkenazi, Ganov, 1981) are due to the fact that those data were obtained with the use of elasticity characteristics in the direction at the angle of $45^{\circ}$ to principal anisotropy axes transverse to reinforcing 


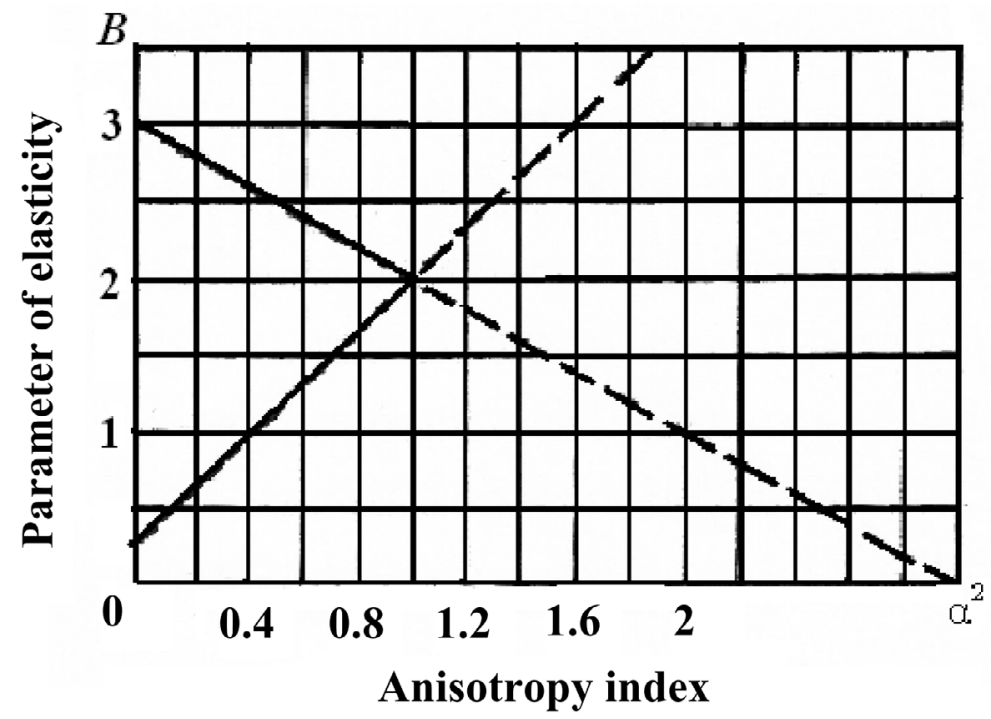

Figure 1. Changes in equation (33) roots depending on $\alpha^{2}$.

fibers. According to the authors (Glukhikh, 2001), the method for experimental determination of these elasticity characteristics is imperfect.

We obtained results corresponding to relations between integration constants in differential equations (6)-(8). However, even with account for the fact that the methods for determination of elastic constants is imperfect, our calculations in most cases are quite close to the available data. In the example considered, this holds for the value of the equation root $B=3-\alpha^{2}$.

It was theoretically confirmed that for an anisotropic body the value $B$ can differ from 2 upward or downward (excluding $B=2$ for an isotropic body).

With account for root $(34$, a) of algebraic equation (33), differential equation in polar coordinates (1) will take the following form (Glukhikh, 1998):

$$
\begin{aligned}
& \frac{\partial^{4} F}{\partial r^{4}}+\frac{1+5 \alpha^{2}}{3} \cdot \frac{1}{r^{2}} \cdot \frac{\partial^{4} F}{\partial r^{2} \partial \theta^{2}}+\frac{\alpha^{2}}{r^{4}} \cdot \frac{\partial^{4} F}{\partial \theta^{4}}+\frac{2}{r} . \\
& \frac{\partial^{3} F}{\partial r^{3}}-\frac{1+5 \alpha^{2}}{3} \cdot \frac{1}{r^{3}} \cdot \frac{\partial^{3} F}{\partial r \partial \theta^{2}}-\frac{\alpha^{2}}{r^{2}} \cdot \frac{\partial^{2} F}{\partial r^{2}}+ \\
& +\frac{1+11 \alpha^{2}}{3} \cdot \frac{1}{r^{4}} \cdot \frac{\partial^{2} F}{\partial \theta^{2}}+\frac{\alpha^{2}}{r^{2}} \cdot \frac{\partial F}{\partial r}=0
\end{aligned}
$$

A similar solution (not given in the article) can be performed for a plane problem in displacements, using the differential equations obtained earlier.

The differential equations given above allow solving tasks related to cylindrically anisotropic orthotropic bodies, in which correlations between elastic constants in principal anisotropy directions, that are affected by roots $(34, a)$ and $(34, b)$ of algebraic equation (33).

For composites, in which the correlation between elastic constants in principal anisotropy directions is affected by law (37), differential equations can be written in the following form:

- in Cartesian coordinates:

$$
\begin{aligned}
& \frac{\partial^{4} F}{\partial x^{4}}\left(x^{4}+\frac{1+5 \alpha^{2}}{3} x^{2} y^{2}+\alpha^{2} y^{4}\right)+\frac{\partial^{4} F}{\partial y^{4}} . \\
& \cdot\left(y^{4}+\frac{1+5 \alpha^{2}}{3} x^{2} y^{2}+\alpha^{2} x^{4}\right)+2 \frac{\partial^{4} F}{\partial y \partial x^{3}} \text {. } \\
& {\left[2 x^{2}+\frac{1+5 \alpha^{2}}{3}\left(y^{2}-x^{2}\right)-2 \alpha^{2} y^{2}\right] \cdot x y+2 \frac{\partial^{4} F}{\partial x \partial y^{3}} \text {. }} \\
& {\left[2 y^{2}+\frac{1+5 \alpha^{2}}{3}\left(x^{2}-y^{2}\right)-2 \alpha^{2} x^{2}\right] \cdot x y+\frac{\partial^{4} F}{\partial x^{2} \partial y^{2}} .} \\
& {\left[6 x^{2} y^{2}+\frac{1+5 \alpha^{2}}{3}\left(x^{4}-4 x^{2} y^{2}+y^{4}\right)+6 \alpha^{2} x^{2} y^{2}\right]+\frac{\partial^{3} F}{\partial x^{3}} \text {. }} \\
& \cdot\left[2 x^{2}+\frac{1+5 \alpha^{2}}{3}\left(3 y^{2}-x^{2}\right)-6 \alpha^{2} y^{2}\right] \cdot x+\frac{\partial^{3} F}{\partial y^{3}} \text {. } \\
& {\left[2 y^{2}+\frac{1+5 \alpha^{2}}{3}\left(3 x^{2}-y^{2}\right)-6 \alpha^{2} x^{2}\right] \cdot y+3 \frac{\partial^{3} F}{\partial y \partial x^{2}} \text {. }} \\
& {\left[2 x^{2}\left(1+2 \alpha^{2}\right)+\frac{1+5 \alpha^{2}}{3}\left(y^{2}-3 x^{2}\right)-2 \alpha^{2} y^{2}\right] \cdot y+3 \frac{\partial^{3} F}{\partial x \partial y^{2}} \text {. }} \\
& {\left[2 y^{2}\left(1+2 \alpha^{2}\right)+\frac{1+5 \alpha^{2}}{3}\left(x^{2}-3 y^{2}\right)-2 \alpha^{2} x^{2}\right] \cdot x+\frac{\partial^{2} F}{\partial x^{2}} \text {. }} \\
& \frac{\alpha^{2}-1}{3} \cdot\left(x^{2}-y^{2}\right)+\frac{\partial^{2} F}{\partial y^{2}} \cdot \frac{1-\alpha^{2}}{3} \cdot\left(x^{2}-y^{2}\right)+ \\
& +\frac{4 \partial^{2} F}{3 \partial x \partial y} \cdot\left(\alpha^{2}-1\right) \cdot x y=0
\end{aligned}
$$

- in polar coordinates (Glukhikh, 1998):

$\frac{\partial^{4} F}{\partial r^{4}}+\left(3-\alpha^{2}\right) \cdot \frac{1}{r^{2}} \cdot \frac{\partial^{4} F}{\partial r^{2} \partial \theta^{2}}+\frac{\alpha^{2}}{r^{4}} \cdot \frac{\partial^{4} F}{\partial \theta^{4}}+\frac{2}{r}$.

$\cdot \frac{\partial^{3} F}{\partial r^{3}}-\left(3-\alpha^{2}\right) \cdot \frac{1}{r^{3}} \cdot \frac{\partial^{3} F}{\partial r \partial \theta^{2}}-\frac{\theta^{2}}{r^{2}} \cdot \frac{\partial^{2} F}{\partial r^{2}}+$ 
$+\left(3+\alpha^{2}\right) \cdot \frac{1}{r^{4}} \cdot \frac{\partial^{2} F}{\partial \theta^{2}}+\frac{\alpha^{2}}{r^{3}} \cdot \frac{\partial F}{\partial r}=0$

- in Cartesian coordinates:

$$
\begin{aligned}
& \frac{\partial^{4} F}{\partial x^{4}}\left[x^{2}\left(x^{2}+3 y^{2}\right)+\alpha^{2} y^{2}\left(y^{2}-x^{2}\right)\right]+\frac{\partial^{4} F}{\partial y^{4}} \\
& \cdot\left[y^{2}\left(y^{2}+3 x^{2}\right)+\alpha^{2} x^{2}\left(x^{2}-y^{2}\right)\right]+2 \frac{\partial^{4} F}{\partial x^{3} \partial y}\left(3 y^{2}-x^{2}\right) \cdot\left(1-\alpha^{2}\right) \\
& \cdot x y+2 \frac{\partial^{4} F}{\partial x \partial y^{3}} \cdot\left(3 x^{2}-y^{2}\right)\left(1-\alpha^{2}\right) \cdot x y+\frac{\partial^{4} F}{\partial x^{2} \partial y^{2}} \\
& \cdot\left[2\left(5 \alpha^{2}-3\right) x^{2} y^{2}+\left(3-\alpha^{2}\right)\left(x^{4}+y^{4}\right)\right]+\frac{\partial^{3} F}{\partial x^{3}}\left(1-\alpha^{2}\right)\left(9 y^{2}-x^{2}\right) \cdot x+ \\
& +\frac{\partial^{3} F}{\partial y^{3}}\left(1-\alpha^{2}\right)\left(9 x^{2}-y^{2}\right) \cdot y+3 \frac{\partial^{3} F}{\partial y \partial x^{2}}\left(1-\alpha^{2}\right)\left(3 y^{2}-7 x^{2}\right) \cdot y+ \\
& +3 \frac{\partial^{3} F}{\partial x \partial y^{2}}\left(1-\alpha^{2}\right)\left(3 x^{2}-7 y^{2}\right) \cdot x+3 \frac{\partial^{2} F}{\partial x^{2}}\left(\alpha^{2}-1\right)\left(x^{2}-y^{2}\right)+ \\
& +3 \frac{\partial^{2} F}{\partial y^{2}}\left(1-\alpha^{2}\right)\left(x^{2}-y^{2}\right)+12 \frac{\partial^{2} F}{\partial y \partial x}\left(\alpha^{2}-1\right) \cdot x y=0
\end{aligned}
$$

The full set of characteristics of elastic stress-strain behavior of orthotropic materials includes nine independent elastic constants subject to experimental determination. When determining deformation in an orthotropic material in the direction not coinciding with principal anisotropy axes, values of elastic constants in an arbitrary direction are required. Equations simultaneously including different elastic constants (Ashkenazi, 1978; Ashkenazi, Ganov, 1981; Lekhnitsky, 1957; Rabinovich, 1946) are required for calculation purposes:

$$
\begin{aligned}
& \frac{1}{E_{x^{\prime}}}=\frac{n_{1}^{4}}{E_{x}}+\frac{l_{1}^{4}}{E_{y}}+\frac{m_{1}^{4}}{E_{z}}+\left(\frac{1}{G_{x y}}-\frac{2 \mu_{x y}}{E_{x}}\right) n_{1}^{2} l_{1}^{2}+ \\
& +\left(\frac{1}{G_{y z}}-\frac{2 \mu_{y z}}{E_{y}}\right) l_{1}^{2} m_{1}^{2}+\left(\frac{1}{G_{z x}}-\frac{2 \mu_{z x}}{E_{z}}\right) m_{1}^{2} n_{1}^{2} \\
& \frac{1}{G_{x^{\prime} y^{\prime}}}=4\left(\frac{n_{1}^{2} n_{2}^{2}}{E_{x}}+\frac{l_{1}^{2} l_{2}^{2}}{E_{y}}+\frac{m_{1}^{2} m_{2}^{2}}{E_{z}}\right)-8 . \\
& \cdot\left(\frac{\mu_{x y}}{E_{x}} n_{1} n_{2} l_{1} l_{2}+\frac{\mu_{y z}}{E_{y}} l_{1} l_{2} m_{1} m_{2}+\frac{\mu_{z x}}{E_{z}} m_{1} m_{2} n_{1} n_{2}\right)+ \\
& +\frac{\left(n_{1} l_{2}+l_{1} n_{2}\right)^{2}}{G_{x y}}+\frac{\left(l_{1} m_{2}+m_{1} l_{2}\right)^{2}}{G_{y z}}+\frac{\left(m_{1} n_{2}+n_{1} m_{2}\right)^{2}}{G_{z x}} \\
& \frac{\mu_{x^{\prime} y^{\prime}}}{E_{x^{\prime}}}=-\frac{n_{1}^{2} n_{2}^{2}}{E_{x}}-\frac{l_{1}^{2} l_{2}^{2}}{E_{y}}-\frac{m_{1}^{2} m_{2}^{2}}{E_{z}}+\frac{\mu_{x y}}{E_{x}}\left(n_{1}^{2} l_{2}^{2}+n_{2}^{2} l_{1}^{2}\right)+ \\
& \frac{\mu_{y z}}{E_{y}}\left(l_{1}^{2} m_{2}^{2}+l_{2}^{2} m_{1}^{2}\right)+\frac{\mu_{z x}}{E_{z}}\left(m_{1}^{2} n_{2}^{2}+m_{2}^{2} n_{1}^{2}\right)- \\
& -\frac{n_{1} n_{2} l_{1} l_{2}}{G_{x y}}-\frac{l_{1} l_{2} m_{1} m_{2}}{G_{y z}}-\frac{m_{1} m_{2} n_{1} n_{2}}{G_{z x}}
\end{aligned}
$$$$
\text { where } n_{1}, n_{2}, \ldots m_{1}, m_{2} \text { - directional cosines. }
$$

Considering correlations (32) between elastic constants, equation (42) can be written for a cylindrically anisotropic orthotropic body in cylindrical coordinates:

$$
\begin{aligned}
& \frac{1}{E_{x^{\prime}}}=\frac{n_{1}^{4}}{E_{r}}+\frac{l_{1}^{4}}{E_{t}}+\frac{m_{1}^{4}}{E_{a}}+\frac{3-\alpha^{2}}{E_{t}} n_{1}^{2} l_{1}^{2}+\left(\frac{1}{G_{t a}}-\frac{2 \mu_{t a}}{E_{t}}\right) . \\
& \cdot l_{1}^{2} m_{1}^{2}+\left(\frac{1}{G_{a r}}-\frac{2 \mu_{a r}}{E_{a}}\right) m_{1}^{2} n_{1}^{2}
\end{aligned}
$$

- for the case when $B_{(2)}=3-\alpha^{2}$.

For an anisotropic material, in which elastic constants satisfy root (34), equation (45) takes the following form:

$$
\begin{aligned}
& \frac{1}{E_{x^{\prime}}}=\frac{n_{1}^{4}}{E_{r}}+\frac{l_{1}^{4}}{E_{t}}+\frac{m_{1}^{4}}{E_{a}}+\frac{1+5 \alpha^{2}}{3 E_{t}} n_{1}^{2} l_{1}^{2}+ \\
& +\left(\frac{1}{G_{t a}}-\frac{2 \mu_{t a}}{E_{t}}\right) l_{1}^{2} m_{1}^{2}+\left(\frac{1}{G_{a r}}-\frac{2 \mu_{a r}}{E_{a}}\right) m_{1}^{2} n_{1}^{2}
\end{aligned}
$$

Equations allowing determining the Poisson's ratios with account for new data $(34, a, b)$ regarding elastic constants of a material with curvilinear anisotropy are as follows:

- for the anisotropy plane passing through axes $X Y$ :

$\mu_{r t}=\frac{1}{2}\left[\frac{E_{r}}{E_{t}}+\frac{E_{r}}{G_{r t}}+1-\frac{4 E_{r}}{E_{r t}^{(45)}}\right]$

where $E_{t} / E_{r}=\alpha^{2} ; G_{r t}^{\mathrm{I}}=\frac{3 E_{t}}{1+5 \alpha^{2}+6 \mu_{t r}}$ from (36); $G_{r t}^{\mathrm{II}}=\frac{E_{t}}{3-\alpha^{2}+2 \mu_{t r}}$ from (37);

$E_{r t}^{(45)}=E_{t}$ from (45). Substituting (36), (37) and $E_{r t}^{(45)}$ to (47) and after rearrangements, the following is obtained for both options of a cylindrically orthotropic material:

$\mu_{r t}=\frac{\mu_{r t}}{\alpha^{2}}$

The obtained equation is in good agreement with the experimental data (Ashkenazi, Ganov, 1981).

- in two other (longitudinal) planes:

$\mu_{t a}=\frac{1}{2}\left[\frac{E_{t}}{E_{a}}+\frac{E_{t}}{G_{t a}}+1-\frac{4 E_{t}}{E_{t a}^{(45)}}\right]$
$\mu_{a r}=\frac{1}{2}\left[\frac{E_{a}}{E_{r}}+\frac{E_{a}}{G_{a r}}+1-\frac{4 E_{a}}{E_{a r}^{(45)}}\right]$

For the shear modulus upon rotation of axes in polar coordinates in the anisotropy plane perpendicular to the longitudinal axis with the use of (36): 
$\frac{1}{G_{x^{\prime} y^{\prime}}}=4\left(\frac{n_{1}^{2} n_{2}^{2}}{E_{r}}+\frac{l_{1}^{2} l_{2}^{2}}{E_{t}}+\frac{m_{1}^{2} m_{2}^{2}}{E_{a}}\right)-8\left(\frac{\mu_{r t}}{E_{r}} n_{1} n_{2} l_{1} l_{2}+\frac{\mu_{t a}}{E_{t}} l_{1} l_{2} m_{1} m_{2}+\frac{\mu_{a r}}{E_{a}} m_{1} m_{2} n_{1} n_{2}\right)+$ $+\frac{\left(n_{1} l_{2}+l_{1} n_{2}\right)^{2}}{3 E_{t}}\left(1+5 \alpha^{2}+6 \mu_{t r}\right)+\frac{\left(l_{1} m_{2}+l_{2} m_{1}\right)^{2}}{G_{t a}}+\frac{\left(m_{1} n_{2}+m_{2} n_{1}\right)^{2}}{G_{a r}}$

the same, with the use of (37):

$\frac{1}{G_{x^{\prime} y^{\prime}}}=4\left(\frac{n_{1}^{2} n_{2}^{2}}{E_{r}}+\frac{l_{1}^{2} l_{2}^{2}}{E_{t}}+\frac{m_{1}^{2} m_{2}^{2}}{E_{a}}\right)-8\left(\frac{\mu_{r t}}{E_{r}} n_{1} n_{2} l_{1} l_{2}+\frac{\mu_{t a}}{E_{t}} l_{1} l_{2} m_{1} m_{2}+\frac{\mu_{a r}}{E_{a}} m_{1} m_{2} n_{1} n_{2}\right)+$
$+\frac{\left(n_{1} l_{2}+l_{1} n_{2}\right)^{2}}{E_{t}}\left(3-\alpha^{2}+2 \mu_{t r}\right)+\frac{\left(l_{1} m_{2}+l_{2} m_{1}\right)^{2}}{G_{t a}}+\frac{\left(m_{1} n_{2}+m_{2} n_{1}\right)^{2}}{G_{a r}}$

Thus, upon transition from radial to tangential direction, the elastic modulus changes from the value $E_{r}$ to $E_{t}$, taking at $\theta=45$ the value $E_{t}$, subsequently passing through the minimum $E_{\text {min }}$.

Such rather simple method to determine the value of the elastic modulus $E_{x y}^{(45)}$ opens wide possibilities for application of equations obtained by Ye.K. Ashkenazi, A.N. Mitinsky, S. G. Lekhnitsky, with the purpose to calculate other elastic constants.

Excluding the Poisson's ratio from these equations, we obtain the following dependencies:

$E_{x^{\prime}}=E_{t} /\left[\alpha^{2} \operatorname{Cos}^{4} \theta+\operatorname{Sin}^{4} \theta+\left(\frac{4 E_{t}}{E_{x y}^{(45)}}-\alpha^{2}-1\right) \operatorname{Cos}^{2} \theta \operatorname{Sin}^{2} \theta\right]$

$\frac{1}{G_{x^{\prime} y^{\prime}}}=\frac{\operatorname{Cos}^{2} 2 \theta}{G_{r t}}+\frac{\operatorname{Sin}^{2} 2 \theta}{G_{x y}^{(45)}}$

The elastic modulus $E_{x y}^{(45)}$ can also be calculated using equations (53), (45).

Equating the terms in round brackets in equations (45) and (53), we obtain the following:

$\frac{1}{G_{r t}}-\frac{2 \mu_{r t}}{E_{r}}=\frac{4}{E_{x y}^{(45)}}-\frac{1}{E_{r}}-\frac{1}{E_{t}}$

where $\frac{1}{G_{r t}}-\frac{2 \mu_{r t}}{E_{r}}=\frac{B}{E_{t}}$ or $B=\frac{E_{t}}{G_{r t}}-2 \alpha^{2} \mu_{r t}$

The last correlation interconnects elastic constants in the plane $X Y$ for a cylindrically anisotropic body. Upon solving differential equation (8) in polynomials, this correlation takes the value satisfying the differential equation:

$B=3-\alpha^{2}$

Then, $\frac{3-\alpha^{2}}{E_{t}}=\frac{4}{E_{x y}^{(45)}}-\frac{1}{E_{r}}-\frac{1}{E_{t}}$

After rearrangements, the following is obtained:

$E_{x y}^{(45)}=E_{t}$

Equation (52) for the elastic modulus will have the following form:
$\frac{1}{E_{x^{\prime}}}=\frac{\operatorname{Cos}^{4} \theta}{E_{r}}+\frac{\operatorname{Sin}^{4} \theta}{E_{t}}+\frac{3-\alpha^{2}}{E_{t}} \operatorname{Cos}^{2} \theta \operatorname{Sin}^{2} \theta$

- for the Poisson's ratio:

$\mu_{x^{\prime} y^{\prime}}=-E_{x^{\prime}}\left[\frac{2\left(\alpha^{2}-1\right)}{E_{t}} \operatorname{Sin}^{2} \theta \operatorname{Cos}^{2} \theta-\frac{\mu_{r t}}{E_{r}}\right]$

The first two extreme values (at $\theta=0^{\circ}$ and $\theta=\pi / 2$ ) correspond to the principal anisotropy planes. The third value can be found by equating the multiplier in square brackets to zero:

$-2 \alpha^{2} \operatorname{Cos}^{2} \theta+2 \operatorname{Sin}^{2} \theta+\left(3-\alpha^{2}\right)\left(\operatorname{Cos}^{2} \theta-\operatorname{Sin}^{2} \theta\right)=0$

After rearrangement of the last equation, the following is obtained:

$3 \operatorname{Cos}^{2} \theta-\operatorname{Sin}^{2} \theta=0$

wherefrom the value of angle $\theta: \theta=\operatorname{arctg} \sqrt{3}=60^{\circ}$

It should be noted that the abscissa of extremum point does not depend from the type of material and the angle $\theta$ equals $60^{\circ}$.

The extreme value of the elastic modulus corresponding to this angle is as follows:

$E_{x^{\prime}}=E_{\min }=8 E_{t} /\left(9-\alpha^{2}\right)$

As per the calculation results, it can be concluded that the elastic modulus reaches its minimum value when the slope angle of the growth ring relative to the board face (as an example, wood is taken) equals $30^{\circ}$.

It is interesting that, at $\theta=45^{\circ}$, the transverse elastic modulus has the value equal to Et.

With account for $B=3-\alpha^{2}$, the shear modulus can be calculated using the equation obtained after rearrangement of (54):

$\frac{1}{G_{x^{\prime} y^{\prime}}}=\frac{8\left(\alpha^{2}-1\right)}{E_{t}} \operatorname{Sin}^{2} \theta \cos ^{2} \theta+\frac{1}{G_{r t}}$

Considering that $B=3-\alpha^{2}$, and, on the other side, $B=\frac{E_{t}}{G_{t}}-\frac{2 \mu_{t} E_{t}}{E_{r}}$ we will obtain the following:

$3-\alpha^{2}=\frac{E_{t}}{G_{r t}}-\frac{2 \mu_{r t} E_{t}}{E_{r}}$ or $G_{r t}=\frac{E_{t}}{3-\alpha^{2}+2 \mu_{t r}}$ form:

Considering (63), equation (62) will take the following $\frac{1}{G_{x^{\prime} y^{\prime}}}=\frac{8\left(\alpha^{2}-1\right)}{E_{t}} \operatorname{Sin}^{2} \theta \operatorname{Cos}^{2} \theta+\frac{3-\alpha^{2}+2 \mu_{t r}}{E_{t}}$

For the shear modulus upon rotation of axes, we will obtain the following equation: 


$$
G_{x^{\prime} y^{\prime}}=\frac{E_{t}}{2\left(\alpha^{2}-1\right) \operatorname{Sin}^{2} 2 \theta+3-\alpha^{2}+2 \mu_{t r}}
$$

Let us analyze the obtained function (65) to find the extreme value, i.e. $\frac{d}{d \theta}\left(G_{x^{\prime} y^{\prime}}\right)=0$, and then we will get the following equation:

$4\left(\alpha^{2}-1\right) E_{t} \operatorname{Sin} 4 \theta=0$

whence it follows that its left part becomes zero in case when $\sin 4 \theta$ is equal to zero, i.e. at $\theta=0, \theta=45^{\circ}$ and $\theta=90^{\circ}$.

At $\theta=0$ and $\theta=90^{\circ}$ we will get the same values: $G_{x^{\prime} y^{\prime}}=G_{r}$.

At $\theta=45^{\circ}$ the shear modulus will have the maximum value:

$G_{x^{\prime} y^{\prime}}^{(45)}=G_{45}=\frac{E_{t}}{\alpha^{2}+1+2 \mu_{t r}}$

After rearrangements, equation (67) will coincide with equation (31) as in (Ashkenazi, Ganov, 1981; Ashkenazi, 1978, Lekhnitsky, 1957).

To calculate the Poisson's ratio $\mu_{x^{\prime} y^{\prime \prime}}$ let us use equation (59) and substitute the following in it:

$\frac{1}{G_{x y}}-\frac{2 \mu_{x y}}{E_{x}}=\frac{1}{G_{r t}}-\frac{2 \mu_{r t}}{E_{r}}=\frac{3-\alpha^{2}}{E_{t}}$

$\mu_{x^{\prime} y^{\prime}}=\frac{2\left(1-\alpha^{2}\right) \operatorname{Sin}^{2} \theta \operatorname{Cos}^{2} \theta-\frac{3-\alpha^{2}}{2}+\frac{E_{t}}{2 G_{r t}}}{\alpha^{2} \operatorname{Cos}^{4} \theta+\left(3-\alpha^{2}\right) \sin ^{2} \theta \operatorname{Cos}^{2} \theta+\operatorname{Sin}^{4} \theta}$

- for the angle $\theta=45^{\circ}$ :

$\mu_{x^{\prime} y^{\prime}}^{(45)}=\frac{E_{t}}{2 G_{r t}}-1$

Theoretically, at some values of $E_{\text {and }}$ and $G_{r}$, the positive value of the Poisson's ratio $\mu_{x^{\prime} y^{\prime}}^{(45 f}$ can be obtained from (69), which was observed by Ye.K. Ashkenazi in experiments.

Let us demonstrate the process of calculating the Poisson's ratio $\mu_{x^{\prime} y}$ using the existing experimental data $E_{t}, \alpha^{2}$ (Ashkenazi, Ganov, 1981) for natural composites with an example.

\section{Example 1}

It is required to calculate the Poisson's ratios for pine at $E_{t}=582 \mathrm{MPa}, \alpha^{2}=0.5178$.

The calculated value $G_{r t}$ as per equation (53) is as follows:

$$
\begin{aligned}
& G_{r t}=187.6 \mathrm{MPa} . \\
& \text { Then: } \\
& \theta=0^{\circ} ; \mu_{x^{\prime} y^{\prime}}=\frac{-3+0.5178+582 / 187.6}{2 \cdot 0.5178}=0.5980 \\
& \theta=90^{\circ} ; \mu_{x^{\prime} y^{\prime}}=\frac{-3+0.5178+582 / 187.6}{2}=0.31
\end{aligned}
$$

$$
\begin{aligned}
\theta & =45^{\circ} ; \\
\mu_{x^{\prime} y^{\prime}}^{(45)} & =\frac{2(1-0.5178) \cdot 0,25-\frac{3-0.5178}{2}+\frac{582}{2 \cdot 187.6}}{0.5178 \cdot 0.25+(3-0.5178) \cdot 0.25+0.25}=0.553
\end{aligned}
$$

As per the invariant relation $E_{t} / E_{r}=\mu_{t r} / \mu_{r t}$, which is known in the task involving wood $\left(\alpha^{2}=0.5178\right)$, if we take one of the Poisson's ratios according to the experimental data (Ashkenazi, Ganov, 1981), e.g. $\mu_{t r}=0.31$, then $\mu_{r t}=$ 0.5987 (the empirical value as per (Ashkenazi, Ganov, 1981): $\mu_{r t}=0.64$ ) will be obtained. On the contrary, at $\mu_{r t}=$ 0.64 , the value $\mu_{r t}=0.33$ will be obtained, which is quite close to the empirical value.

\section{Example 2}

It is required to calculate the Poisson's ratios for spruce at $E_{t}=400 \mathrm{MPa}$

$$
G_{r t}=136.5 \mathrm{MPa}
$$

$\alpha^{2}=0.5706$

$\mu_{x^{\prime} y^{\prime}}=\mu_{t}=-E_{x}\left[\left(\frac{\alpha^{2}}{E_{t}}+\frac{1}{E_{t}}-\frac{3-\alpha^{2}}{E_{t}}\right) \operatorname{Sin}^{2} \theta \operatorname{Cos}^{2} \theta+\frac{3-\alpha^{2}}{2 E_{t}}-\frac{1}{2 G_{t}}\right]=$

$=\frac{-E_{t}}{\alpha^{2} \operatorname{Cos}^{4} \theta+\operatorname{Sin}^{4} \theta+\left(3-\alpha^{2}\right) \cos ^{2} \theta \operatorname{Sin}^{2} \theta} \cdot\left[\frac{2\left(\alpha^{2}-1\right) \operatorname{Sin}^{2} \theta \operatorname{Cos}^{2} \theta}{E_{t}}+\frac{3-\alpha^{2}}{2 E_{t}}-\frac{1}{2 G_{t}}\right]=$

$=-\frac{3-\alpha^{2}}{2 \alpha^{2}}+\frac{E_{t}}{2 G_{r t} \alpha^{2}}=-\frac{3-0.5706}{2 \cdot 0.5706}+\frac{400}{2 \cdot 136.5 \cdot 0.5706}=0.439$

$\mu_{r t}=0.439$ The table value of $\mu_{r t}=0.42$

$\mu_{t r}=0.439 \cdot \alpha^{2}=0.439 \cdot 0.5706=0.2505$

The table value of $\mu_{r t}=0.25$.

Correlations based on the requirement for the positive value of elastic potential are as follows:

$\mu_{a}<\sqrt{E_{a} / E_{r}} ; \mu_{t a}<\sqrt{E_{t} / E_{a}} ; \mu_{r t}<\sqrt{E_{r} / E_{t}}$

Fulfillment of the following correlation for an orthotropic body is checked (Ashkenazi, Ganov, 1981):

$$
\begin{aligned}
& \frac{2}{E_{r}}+\frac{2}{E_{t}}+\frac{1}{G_{r t}}=\frac{4}{E_{x y}^{(45)}}+\frac{1}{G_{45}} \\
& \frac{2 \alpha^{2}}{E_{t}}+\frac{2}{E_{t}}+\frac{3-\alpha^{2}+2 \mu_{t r}}{E_{t}}=\frac{4}{E_{t}}+\frac{\alpha^{2}+1+2 \mu_{t r}}{E_{t}} \\
& \frac{2 \alpha^{2}+2+3-\alpha^{2}+2 \mu_{t r}}{E_{t}}=\frac{5+\alpha^{2}+2 \mu_{t r}}{E_{t}}
\end{aligned}
$$

The correlation is observed, the left and right parts are identically equal.

The same correlation is observed for pine wood:

$$
\frac{2}{1124}+\frac{2}{582}+\frac{1}{187.6}=\frac{4}{582}+\frac{1}{272.2}
$$

The value of the directional cosine determining the position of axis $X^{\prime}$, relative to which the function of the elastic modulus $E_{x^{\prime}}$ takes the extreme value (in the $X Y$ 
Table 2. Elastic constants for several wood species.

\begin{tabular}{|c|c|c|c|c|c|c|c|c|c|c|}
\hline \multirow[t]{2}{*}{ Wood specie } & \multirow[t]{2}{*}{$E_{t}, \mathrm{MPa}$} & \multirow[t]{2}{*}{$\begin{array}{l}G_{r t} \\
\mathrm{MPa}\end{array}$} & \multirow[t]{2}{*}{$a^{2}=E_{t} / E_{r}$} & \multicolumn{7}{|c|}{$\begin{array}{l}\text { Poisson's ratio as per equation (69) at the following slope angles of the } \\
\text { growth ring }\end{array}$} \\
\hline & & & & $\theta=0^{\circ}$ & $15^{\circ}$ & $30^{\circ}$ & $45^{\circ}$ & $60^{\circ}$ & $75^{\circ}$ & $90^{\circ}$ \\
\hline Pine & 582 & 187.6 & 0.5178 & $\frac{0.6}{0.64^{*}}$ & 0.6068 & 0.59925 & 0.553 & 0.463 & 0.3646 & $\frac{0.31}{0.31^{*}}$ \\
\hline Spruce & 400 & $\frac{136.5}{347^{*}}$ & 0.5706 & $\frac{0.44}{0.42^{*}}$ & 0.4658 & 0.4905 & 0.4652 & 0.3905 & 0.2962 & $\frac{0.25}{0.25^{*}}$ \\
\hline Beech & 1,160 & $\frac{361.1}{467^{*}}$ & 0.5077 & $\frac{0.71}{0.75^{*}}$ & 0.7 & 0.668 & 0.596 & 0.513 & 0.4098 & $\underline{0.36}$ \\
\hline Oak & 985 & $\frac{312.8}{403^{*}}$ & 0.4508 & $\frac{0.665}{0.64^{*}}$ & 0.6689 & 0.6412 & 0.575 & 0.4766 & 0.36 & $\frac{0.30}{0.30^{*}}$ \\
\hline Ash & 818 & $\frac{256.6}{284^{*}}$ & 0.5322 & $\frac{0.6765}{0.71^{*}}$ & 0.6728 & 0.6493 & 0.594 & 0.50586 & 0.4074 & $\underline{0.36}$ \\
\hline Fir & 490 & $\frac{154.1}{150^{*}}$ & 0.5213 & $\frac{0.672}{0.60^{*}}$ & 0.6692 & 0.646 & 0.59 & 0.5 & 0.3993 & $\underline{0.35}$ \\
\hline Maple & 890 & $\frac{275.9}{287^{*}}$ & 0.5742 & $\frac{0.6966}{0.82^{*}}$ & 0.691 & 0.6660 & 0.613 & 0.531 & 0.442 & $\underline{0.4}$ \\
\hline
\end{tabular}

Note: * experimental values (Ashkenazi, Ganov, 1981).

Table 3. Elasticity characteristics of filament-wound fiberglass as per the data presented in some publications (Glukhikh, 2008) and as per the results of author's calculations (given in brackets).

\begin{tabular}{|c|c|c|c|c|}
\hline \multirow{4}{*}{ Elasticity characteristics } & \multicolumn{4}{|c|}{ Filler } \\
\hline & Strand 19 of VM-1 fiber & VM-1 fiber & Strand 19 of VM-1 fiber & VM-1 fiber \\
\hline & \multicolumn{4}{|c|}{ Ratio of fibers } \\
\hline & $n=\infty$ & $n=5$ & $n=2$ & $n=1$ \\
\hline$E_{a}, \mathrm{MPa}$ & 5.7 & 4.76 & 3.68 & 3.09 \\
\hline$E_{r}, \mathrm{MPa}$ & 1.4 & 2.07 & 2.68 & 2.74 \\
\hline$E_{t}, \mathrm{MPa}$ & 1.4 & 1.45 & 1.10 & 1.05 \\
\hline$E_{a r}, \mathrm{MPa}$ & $1.58(1.4)$ & $1.59(2.07)$ & $1.56(2.68)$ & $1.28(2.74)$ \\
\hline$E_{r t}, \mathrm{MPa}$ & $1.4(1.4)$ & $1.26(1.45)$ & $1.19(1.10)$ & $1.07(1.05)$ \\
\hline$E_{t a}, \mathrm{MPa}$ & $1.58(1.4)$ & $1.45(1.45)$ & $1.24(1.10)$ & $1.07(1.05)$ \\
\hline$B^{\prime \prime}$ & 2.3 & 3.77 & 5.15 & 6.7 \\
\hline $\mathrm{G}_{a r}, \mathrm{MPa}$ & $0.575(0.484)$ & $0.531(0.768)$ & $0.505(1.105)$ & $0.396(1.174)$ \\
\hline$B$ & 2 & 2.89 & 3.02 & 2.526 \\
\hline $\mathrm{G}_{r t}, \mathrm{MPa}$ & $0.5(0.5)$ & $0.436(0.526)$ & $0.414(0.373)$ & $0.369(0.358)$ \\
\hline$B^{\prime}$ & 2.3 & 2.7 & 2.25 & 2.62 \\
\hline $\mathrm{G}_{\mathrm{ta}}, \mathrm{MPa}$ & $0.575(0.484)$ & $0.501(0.500)$ & $0.447(0.374)$ & $0.366(0.356)$ \\
\hline$\mu_{\mathrm{ar}}$ & 0.277 & 0.149 & 0.105 & 0.123 \\
\hline$\mu_{\mathrm{ra}}$ & 0.068 & 0.065 & 0.077 & 0.110 \\
\hline$\mu_{r t}$ & 0.4 & 0.325 & 0.431 & 0.417 \\
\hline$\mu_{\mathrm{tr}}$ & 0.4 & 0.227 & 0.177 & 0.160 \\
\hline$\mu_{\mathrm{ta}}$ & 0.068 & 0.099 & 0.105 & 0.151 \\
\hline$\mu_{\text {at }}$ & 0.277 & 0.325 & 0.353 & 0.443 \\
\hline
\end{tabular}


plane at $I_{1}=0$ ), can be found using the following equation (Ashkenazi, 1978):

$l_{1}= \pm \sqrt{\frac{\frac{3}{E_{t}}+\frac{1}{E_{r}}-\frac{4}{E_{x y}^{(45)}}}{\frac{4}{E_{t}}+\frac{4}{E_{r}}-\frac{8}{E_{x y}^{(45)}}}}= \pm \sqrt{\frac{\frac{3}{E_{t}}+\frac{\alpha^{2}}{E_{t}}-\frac{4}{E_{t}}}{E_{t}}+\frac{4 \alpha^{2}}{E_{t}}-\frac{8}{E_{t}}}= \pm \frac{1}{2}$

i.e. $\operatorname{Cos}\left(x, x^{\prime}\right)= \pm \frac{1}{2}$

Thus, the angle between axes $X$ and $X^{\prime}$ is equal to $60^{\circ}$. The same result was obtained above. The second directional cosine:

$m_{1}= \pm \sqrt{1-l_{1}^{2}}= \pm \sqrt{1-\frac{1}{4}}= \pm \frac{\sqrt{3}}{2}$

which corresponds to the angle of $30^{\circ}$.

For equal-reinforced composites $\left(\alpha^{2}=1\right)$, this angle is equal to $45^{\circ}$.

The available standards stipulate for experimental determination of 18 elasticity characteristics $\left(3 E_{i}, 3 G_{i k}, 3 \mu_{i k}, 3 E_{i k}^{(45)}, 3 \mu_{i k}^{(45)}\right)$.

According to the results, the shear moduli are determined using the following equation:

$$
G_{i k}=\frac{E_{i k}^{(45)}}{2\left(1+\mu_{i k}^{(45)}\right)}
$$

As the correlation $\left(B=3-\alpha^{2}\right)$ is established between elastic constants, there is no need in experimental determination of $E_{i k}^{(45)}, \mu_{i k}^{(45)}$. To determine elastic constants, it is sufficient to know their values relative to principal anisotropy axes $\left(3 E_{i} ; 3 \mu_{i k} ; 3 \mu_{k i}\right)$.

Using the equations obtained above, it is possible to determine values of elastic constants relative to any axes position.

If the ratio between elastic constants corresponds to dependency $(34, a)$ for some composites, then equations for the elastic modulus, Poisson's ratio, and shear modulus will be as follows:

$$
\begin{aligned}
& \frac{1}{E_{x^{\prime}}}=\frac{\operatorname{Cos}^{4} \theta}{E_{r}}+\frac{1+5 \alpha^{2}}{3 E_{t}} \operatorname{Sin}^{2} \theta \operatorname{Cos}^{2} \theta+\frac{\operatorname{Sin}^{4} \theta}{E_{t}} \\
& \frac{1}{E_{y^{\prime}}}=\frac{\operatorname{Sin}^{4} \theta}{E_{r}}+\frac{1+5 \alpha^{2}}{3 E_{t}} \operatorname{Sin}^{2} \theta \operatorname{Cos}^{2} \theta+\frac{\operatorname{Cos}^{4} \theta}{E_{t}}
\end{aligned}
$$

$$
\begin{aligned}
& \frac{1}{G_{x^{\prime} y^{\prime}}}=\frac{8\left(1-\alpha^{2}\right)}{3 E_{t}} \operatorname{Sin}^{2} \theta \operatorname{Cos}^{2} \theta+\frac{1}{G_{r t}} \\
& \mu_{x^{\prime} y^{\prime}}=-E_{x^{\prime}}\left[\frac{2\left(1-\alpha^{2}\right)}{3 E_{t}} \operatorname{Sin}^{2} \theta \operatorname{Cos}^{2} \theta-\frac{\mu_{r t}}{E_{r}}\right] \\
& E_{x y}^{(45)}=\frac{3 E_{t}}{1+2 \alpha^{2}}
\end{aligned}
$$

Upon an analysis of function (72) to find the extreme value, the following equation is obtained:

$$
\operatorname{Cos}^{2} \theta+5 \operatorname{Sin}^{2} \theta=0 \text {, }
$$

whence it follows that the elastic modulus $E_{x^{\prime}}$ does not take the third extreme value upon rotation of axes. At $B<2$, composites have only two main modulus values: $E_{r}$ and $E_{t}$.

Analyzing shear modulus function (74) at rotation of axes in the plane of cross-section to find the extreme value, similar extreme values (as at $B=3-\alpha^{2}$ ) are obtained. At other values of the angle $\theta$, shear moduli differ.

Based on equation (75), it is possible to obtain the value of the Poisson's ratio at $\theta=45^{\circ}$ :

$\mu_{x^{\prime} y^{\prime}}^{(45)}=\frac{3 E_{t}}{2\left(1+2 \alpha^{2}\right) G_{r t}}-1$

The Poisson's ratio can be positive if the following correlation is observed:

$$
\frac{E_{t}}{2 G_{r t}}<\frac{1+2 \alpha^{2}}{3}
$$

Comparison of calculated values of elastic constants using the obtained equations with the experimental data for a cylindrically anisotropic body through the example of fiberglass materials (Table 3 ) allows checking the original assumptions underlying the conclusion on their applicability to the examined material. At the $B$ value within the range from 2 to 3 , calculated values of elasticity characteristics are quite close to the experimental ones. At values exceeding 3 , noticeable deviation of the calculated shear modulus $G_{a r}$ from the experimental value is observed. This is due to the fact that at values exceeding 3 , the parameter $B$ does not satisfy differential equation (1). The $B$ value can be affected by inaccuracy in determination of $\mu_{\mathrm{ra}}, G_{a r}$ and imperfection of experiment methods. 


\section{References}

Ashkenazi, Ye.K. (1978). Anizotropiya drevesiny i drevecnyh materialov [Anisotropy of wood and wood materials]. Moscow: Lesnaya Promyshlennost (Forest Industry). (in Russian)

Ashkenazi, Ye.K., Ganov, V.V. (1981). Anizotropiya konstrukcionnyh materialov [Anisotropy of structural materials]. Moscow: Lesnaya Promyshlennost (Forest Industry).(in Russian)

Glukhikh, V.N. (1997). K voprosu o reshenii v polinomah ploskoj zadachi teorii uprugosti dlya pryamougol'noj cilindricheski anizotropnoj polosy [Concerning the solution of the plane problem of the elasticity theory for a rectangular cylindrically anisotropic strip (in polynomials)]. Saint Petersburg: Saint Petersburg State Forest Technical University. (in Russian)

Glukhikh, V.N. (1998). Ploskaya zadacha teorii uprugosti dlya cilindricheski anizotropnogo tela v dekartovyh koordinatah [Plane problem of the elasticity theory for a cylindrically anisotropic body in Cartesian coordinates]. News of the Saint Petersburg State Forest Technical Academy, 6 (164), pp. 141-145. (in Russian)

Glukhikh, V.N. (2001). Primenenie polinomov k resheniyu zadach dlya cilindricheski anizotropnogo tela [Application of polynomials in the solution of problems for a cylindrically anisotropic body]. Saint Petersburg: Saint Petersburg State Forest Technical University. (in Russian)

Glukhikh, V.N. (2007). Uprugie postoyannye cilindricheski anizotropnogo tela [Elastic constants of a cylindrically anisotropic body]. Journal International Academy of Refrigeration, 2. (in Russian)

Glukhikh, V.N. (2008). Svyaz' mezhdu uprugimi postoyannymi cilindricheski anizotropnogo tela [Relations between elastic constants of a cylindrically anisotropic body]. Journal International Academy of Refrigeration, 1. (in Russian)

Glukhikh, V.N. (2010). Differential equations for a cylindrically anisotropic body considering obtained correlations between independent elastic constants. In: Proceedings of the 4th International Eurasian Symposium. Yekaterinburg: Ural State Forest Engineering University, pp. 48-54. (in Russian)

Grigorovich, V.K. (1952). O naivygodnejshem napravlenii volokon v izdeliyah iz anizotropnyh materialov [Concerning the most favorable direction of fibers in products made of anisotropic materials]. Proceedings of the USSR Academy of Sciences, 86 (4), pp. 152-160. (in Russian)

Kuffner, M. (1978). Elastizitatsmodul und Zugfestigkeit von Holz verschiedenen Rohdichte in Abhangigkeit vom Feuchtigkeitgehalt [Modulus of elasticity and breaking strength factor of wood of different true specific density, depending on the moisture content]. Wood as a Raw Material and Goods, 11, pp. 435-440. (in German)

Kurdyumov, N. (1946). Reshenie $v$ polinomah ploskoj zadachi teorii uprugosti [Solution of the plane problem of the elasticity theory (in polynomials)]. PMM, XI. (in Russian)

Lekhnitsky, S.G. (1957). Anizotropnye plastinki [Anisotropic plates]. Moscow: Gostekhizdat. (in Russian)

Lekhnitsky, S.G. (1977). Teoriya uprugosti anizotropnogo tela [Theory of anisotropic body elasticity]. Moscow. (in Russian)

Mitinsky, A.N. (1948). Uprugie postoyannye drevesiny kak ortotropnogo materiala [Elastic constants of wood as an orthotropic material]. Proceedings of the Forest Academy, 63, pp. 22-54. (in Russian)

Mitinsky, A.N. (1949). Torsion and shear moduli of wood as an anisotropic material. Proceedings of the Forest Academy, 65, pp. 49-57. (in Russian)

Rabinovich, A.L. (1946). Ob uprugih postoyannyh i prochnosti anizotropnyh materialov [Concerning elastic constants and strength of anisotropic materials]. Moscow: Buro novoi tekhniki. (in Russian)

Rosato, D.V., Grove, C.S. (1969). Namotka steklonit'yu [Filament winding]. Translated from English. Moscow: Mashinostroyeniye. (in Russian)

Ylinen, A. (1952). Uber die mechanische schaftformtheorie der Baume [On the mechanic theory of the stem form of trees]. Scientific Researches, 6. (in German) 